\title{
Histological evaluation of thyroid lesions using a scanning acoustic microscope
}

\section{Katsutoshi Miura' \\ Hiroyuki Mineta² \\ 'Department of Health Science, Pathology, and Anatomy, ${ }^{2}$ Department of Otorhinolaryngology, Hamamatsu University School of Medicine, \\ Hamamatsu, Japan}

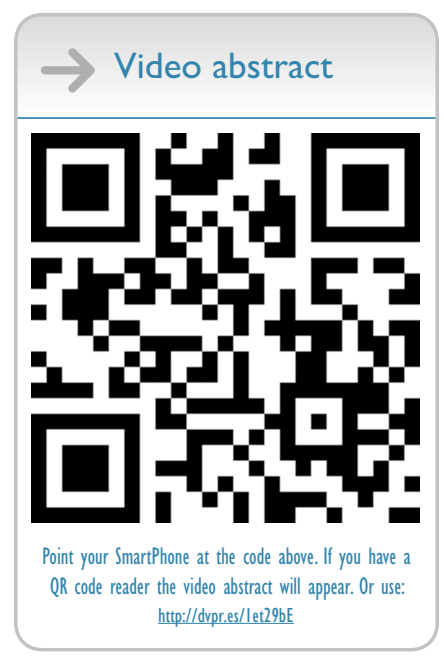

Correspondence: Katsutoshi Miura Department of Health Science, Pathology, and Anatomy, Hamamatsu University School of Medicine, I-20-I Handa-yama, Higashi-ku, Hamamatsu 43I-3192, Japan $\mathrm{Tel}+8 \mathrm{I} 5343528 \mathrm{II}$

Fax +8I 534352800

Email kmiura@hama-med.ac.jp
This article was published in the following Dove Press journal:

Pathology and Laboratory Medicine International

II February 2014

Number of times this article has been viewed

Purpose: A scanning acoustic microscope (SAM) uses an ultrasound to image an object by plotting the speed-of-sound (SOS) through tissues on screen. Because hard tissues result in great SOS, SAM can provide data on the tissue elasticity. This paper investigated the utility of SAM in evaluating thyroid lesions.

Methods: Formalin-fixed, paraffin sections were scanned with a $120 \mathrm{MHz}$ transducer. SOS through each area was calculated and plotted on the screen to provide histological images, and SOS of each lesion was compared and statistically analyzed.

Results: High-concentrated colloids, red blood cells, and collagen fibers showed great SOS, while low-concentrated colloids, parathyroids, lymph follicles, and epithelial tissues including carcinomas demonstrated lower SOS. SAM clearly discriminated structure of thyroid components corresponding to low magnification of light microscopy. Thyroid tumors were classified into three groups by average SOS: the fast group consisted of follicular adenomas/carcinomas and malignant lymphomas; the slow group contained poorly differentiated/undifferentiated carcinomas; and the intermediate group comprised papillary/medullary carcinomas. Fragmented colloids, irregular-shaped follicles, and desmoplastic reactions were observed in the invasive area of surrounding carcinomas.

Conclusion: The SAM imaging method had the following benefits: 1) precise images were acquired in a few minutes without special staining; 2) structural irregularity and desmoplastic reactions, which indicated malignancy, were detected; 3) images reflected tissue elasticity, which was statistically comparable among lesions by SOS; 4) follicular functional activity was predictable by converting colloid concentration to SOS; and 5) tumor classification was predictable by SOS because more poorly differentiated carcinomas had a tendency to show lower SOS.

Keywords: scanning acoustic microscope, tissue elasticity, cancer imaging, thyroid tumor, parathyroid

\section{Introduction}

Thyroid ultrasound (US) is a widespread technique and is used as a first-line diagnostic procedure. ${ }^{1}$ Its usefulness is widely recognized in detecting and characterizing nodular thyroid disease. US equipment with 7-13 MHz frequency transducers can detect 2-3 mm thyroid lesions, but the resolution is not very high and is insufficient for histological diagnosis.

US elastography is a powerful new diagnostic tool that assesses hardness as an indicator of malignancy. ${ }^{1}$ The basic principle of elastography is that tissue compression produces strain (displacement) within the tissues, which is smaller in harder tissues than in softer tissues. Because carcinomas are commonly harder than benign tumors or lesions, detecting nodular elasticity is critical in determining malignancy. 
A scanning acoustic microscope (SAM; Figure 1) uses US to image an object by plotting the speed-of-sound (SOS) through tissues onscreen. ${ }^{2}$ Because harder tissues result in great SOS, SAM can provide data on the elasticity of tissues and lesions. ${ }^{3}$ SAM uses US at $120 \mathrm{MHz}$ with almost the same resolution (approximately $12.5 \mu \mathrm{m}$ ) as the low magnification of a light microscope (LM). The authors recently reported that SAM could be used to generate useful information on the lung, ${ }^{4}$ stomach, ${ }^{5}$ and lymph node ${ }^{6}$ lesions. To date, no reports have been found on the application of SAM in thyroid histology. This report investigated the utility of SAM in evaluating thyroid lesions, including neoplastic and reactive lesions.

\section{Materials and methods}

All histological sections were already diagnosed and prepared from the Hamamatsu University Hospital archives. The research protocol using stored samples from anonymous donors without a link to patient identity was approved by the Research Ethics Committee of Hamamatsu University School of Medicine.

Formalin-fixed, paraffin-embedded blocks were flatsectioned in $10 \mu \mathrm{m}$ thick sections. Because deparaffinized sections repelled water due to surface hydrophobicity and dried out easily, it was necessary to soak sections in distilled water to reduce hydrophobicity. Deparaffinized, unstained sections were soaked in distilled water for at least 3 hours before observation.

\section{Tissue samples}

Inflammatory/nonneoplastic and neoplastic thyroid lesions were selected and examined, ${ }^{7}$ including Hashimoto's

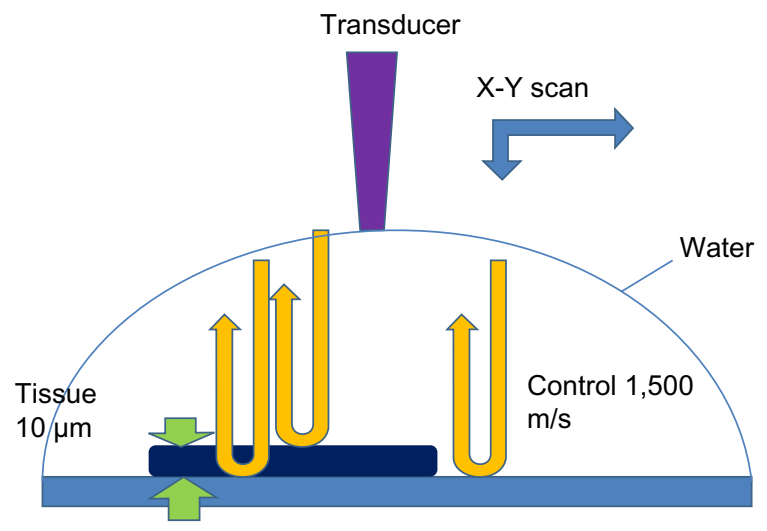

Figure I Design of scanning acoustic microscopy. Ultrasonic waves $(120 \mathrm{MHz})$ from the transducer reflect off both the glass slide and section and return to the transducer. These waves pass through $10 \mu \mathrm{m}$ sample sections with different ultrasonic properties. The transducer automatically scans the section to calculate the speed-ofsound through each area. Distilled water is dropped between the transducer and the section as a coupling fluid. The control speed-of-sound through water is $1,500 \mathrm{~m} / \mathrm{s}$. Abbreviation: $\mathrm{m} / \mathrm{s}$, meter/second. thyroiditis, Graves' disease, papillary carcinoma, follicular carcinoma, medullary carcinoma, undifferentiated carcinoma, follicular adenoma, and primary malignant lymphoma of the thyroid.

Specimens were randomly selected from the computer database of pathological sections and typical lesions of thyroid diseases were identified based on hematoxylin and eosin sections. Because of the necessity of flatness and the $2.4 \mathrm{~mm}^{2}$ limit of calculation areas, hematoxylin and eosinstained sections were examined and observation areas were determined. For a comparative study of lesions, three representative cases from each disease were selected. A blank area on at least one corner was included in each section as the control area.

High-concentrated colloid samples and lymph follicle and fibrous septa samples were collected in patients with Hashimoto's thyroiditis and compressed follicles in those with tumors. Low-concentrated colloid samples were collected from follicles of Graves' disease.

\section{SAM observation}

SAM model AMS-50AI was supplied by Honda Electronics $\mathrm{Co}, \mathrm{Ltd}$, (Toyohashi, Japan) and was equipped with a $120 \mathrm{MHz}$ transducer. SAM functions by directing focused sound from a transducer to a small area of the target object on a glass slide. The sound emitted by an acoustic transducer hits or penetrates the tissues and is reflected on the surface of the tissue or glass. It then returns to the receiver, which is coincident with the transducer. SOS through the tissue is automatically calculated by comparing the time-of-flight of the pulse from the surfaces of both tissue and glass. As controls, SOS of blank areas only through water was determined as 1,500 meter/second $(\mathrm{m} / \mathrm{s}){ }^{8}$

Because the concrete protocol of SAM observation has already been reported, ${ }^{2}$ only an outline is briefly described. To perform SAM imaging, slide sections were placed upside down on the stage above the transducer, and distilled water was added between the transducer and section as a coupling fluid. After mechanical X-Y scanning, SOS from each point on a section was calculated and plotted on the screen to create two-dimensional, color-coded images. The vertical bar on the left and the horizontal bar at the bottom of each SOS figure on the screen indicate the distance $(\mathrm{mm})$ on the slide. The vertical colored column on the right side of the SOS figure on the screen indicates the mean SOS of each square area on the section. From slow to fast, the color of SOS changes gradually from blue to green to yellow to red. The region of interest for acoustic microscopy was determined from the LM images. SOS values at $300 \times 300$ points on 
the screen were calculated and plotted to create the images, and sound data from 64 cross points on the lattice screen were used for statistical analysis.

Each SAM image included a vacant area that counted water only at an SOS of $1,500 \mathrm{~m} / \mathrm{s}$ and a blood area that appeared red in color $(1,750 \mathrm{~m} / \mathrm{s})$ as control areas.

\section{Statistical analysis}

SOS data of thyroid lesions were presented as average \pm standard deviation $(\mathrm{m} / \mathrm{s})$. Student's $t$-tests were used to determine statistically significant differences among the specimens. The level of statistical significance was set at $P<0.01$.

\section{Results}

SAM imaging was obtained by scanning the unstained section within a few minutes. Table 1 and Figure 2 show the average and standard deviation $(\mathrm{m} / \mathrm{s})$ of SOS through thyroid components and lesions. Basic thyroid components, such as thyroid follicles (Figure 3A), interstitial stroma, blood vessels, and parathyroids (Figure 3B), were distinguishable from each other by their own SOS. Parathyroids containing fat cells showed lower SOS, approximately $1,600 \mathrm{~m} / \mathrm{s}$ compared with the surrounding thyroid tissues.

Concentration of colloid contents differed among follicles and functional states. Graves' disease showing hyperthyroidism had elongated follicles in various sizes and displayed an average SOS of approximately $1,570 \mathrm{~m} / \mathrm{s}$ on SAM imaging (Figure 4). The SOS values of colloids in this disease were significantly lower (Figure 2; Table 1) than those of highconcentrated colloids in follicular adenoma (Figure 5A) and Hashimoto's thyroiditis (Figure 5B).

Table I Average and standard deviation $(\mathrm{m} / \mathrm{s})$ of speed-of-sound in thyroid lesions

\begin{tabular}{lll}
\hline & Average & Standard deviation \\
\hline High colloids & $1,814.65$ & 51.05 \\
Low colloids & $1,569.24$ & 47.04 \\
Lymph follicles & $1,595.55$ & 25.78 \\
Parathyroid & $1,598.97$ & 34.39 \\
Blood & $1,746.25$ & 73.27 \\
Fib sept & $1,688.47$ & 53.19 \\
F adenoma & $1,650.32$ & 69.97 \\
Pap Ca & $1,607.65$ & 32.67 \\
F Ca & $1,643.07$ & 62.05 \\
Por Ca & $1,573.81$ & 42.68 \\
Undiff Ca & $1,574.58$ & 29.6 \\
Med Ca & $1,597.22$ & 50.74 \\
Lymphoma & $1,645.12$ & 46.65 \\
\hline
\end{tabular}

Abbreviations: $\mathrm{F}$ adenoma, follicular adenoma; $\mathrm{F}$ carcinoma, follicular carcinoma; Fib sept, fibrous septum; high colloids, concentrated colloid; low colloids, thin colloid; Med Ca, medullary carcinoma; Pap Ca, papillary carcinoma; Por Ca, poorly differentiated carcinoma; Undiff $\mathrm{Ca}$, undifferentiated carcinoma; $\mathrm{m} / \mathrm{s}$, meter/second.
Concentrated proteins, such as blood and dense colloids, and collagen fibers or muscles, such as fibrous septum and vessel walls, displayed great SOS. In contrast, thin colloids, lymph fluids, and loose tissue, such as lymph follicles and parathyroids, had a low SOS. Major thyroid tumors were observed by SAM, and characteristic images were acquired.

Papillary carcinoma consisting of thin elongated cores with branching appeared blue-green in color at approximately $1,600 \mathrm{~m} / \mathrm{s}$ (Figure 6A). Fibrous cores displayed faster yellow-red dots at approximately $1,680 \mathrm{~m} / \mathrm{s}$. Invasive areas with desmoplastic reactions were yellow-green in color (Figure 6B) with greater SOS than papillary carcinomas. The follicular structures at invasive sites were broken with fragmented colloids.

A follicular carcinoma consisting of microfollicles was covered by a fibrous septum and compressed by surrounding normal follicles (Figure 7). The borders between the carcinoma and septum were irregular. Condensed colloids in the follicles were red colored with $>1,750 \mathrm{~m} / \mathrm{s}$ on SAM imaging, while follicles without colloids or pale colloids displayed a green-blue color, corresponding to approximately $1,570 \mathrm{~m} / \mathrm{s}$.

The poorly differentiated carcinomas occupy both morphologically and behaviorally an intermediate position between the differentiated (follicular and papillary carcinomas) and undifferentiated carcinomas. ${ }^{7}$ No large colloids were found in these samples. Carcinoma portions were approximately $1,570 \mathrm{~m} / \mathrm{s}$ in an average SOS (Figure 8), which was slower than the well-differentiated follicular and papillary carcinomas (Table 1; Figure 2).

Undifferentiated carcinomas consisting of spindle cells invaded into the surrounding thyroid follicles (Figure 9). The carcinoma portions were approximately $1,570 \mathrm{~m} / \mathrm{s}$ on SAM imaging. SOS was almost the same in the poorly differentiated carcinomas; however, it was slower than in the well-differentiated carcinomas.

Medullary carcinomas displayed an average SOS of approximately $1,590 \mathrm{~m} / \mathrm{s}$, which was between the poorly/ undifferentiated carcinomas and well-differentiated carcinomas (Figure 10).

Malignant lymphoma of the thyroid commonly arises from Hashimoto's thyroiditis. Several lymphoma cells penetrate thyroid follicles and destroy them (Figure 11). These destructed follicular colloids showed tiny red dots with great SOS. Although lymphocytes themselves displayed a slow SOS (blue-green color), most lymphoma portions showed a faster SOS (yellow-green color) with approximately $1,650 \mathrm{~m} / \mathrm{s}$ on 


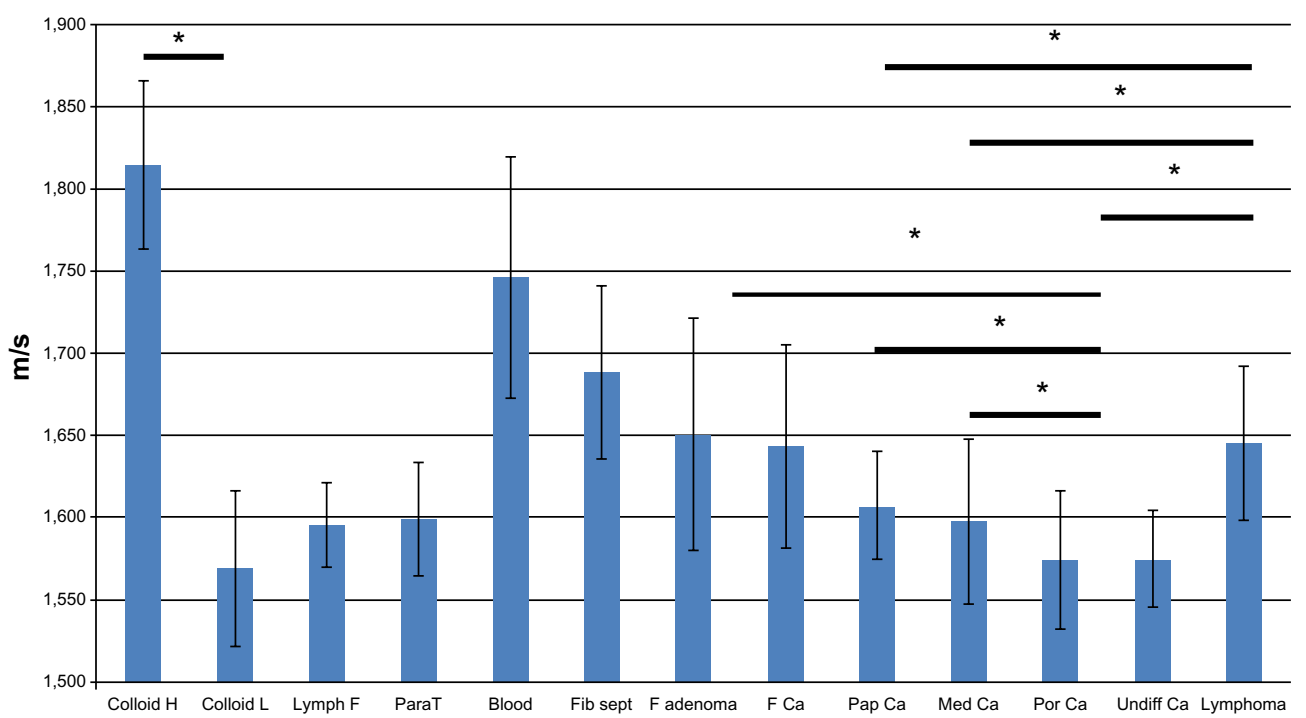

Figure 2 The average \pm standard deviation $(\mathrm{m} / \mathrm{s})$ of speed-of-sound in thyroid lesions. Student's $t$-tests were used to determine statistically significant differences among the specimens.

Note: $* P<0.01$. Horizontal lines indicate significant difference between two values. The bars terminated between two specimens designate that both values have significant difference between the value at the other end.

Abbreviations: Colloid H, high-concentrated colloid; Colloid L, low-concentrated colloid; F adenoma, follicular adenoma; F carcinoma, follicular carcinoma; Fib sept, fibrous septum; Lymph F, lymph follicle; Med Ca, medullary carcinoma; Pap Ca, papillary carcinoma; ParaT, parathyroid; Por Ca, poorly differentiated carcinoma; Undiff Ca, undifferentiated carcinoma; $\mathrm{m} / \mathrm{s}$, meter/second.
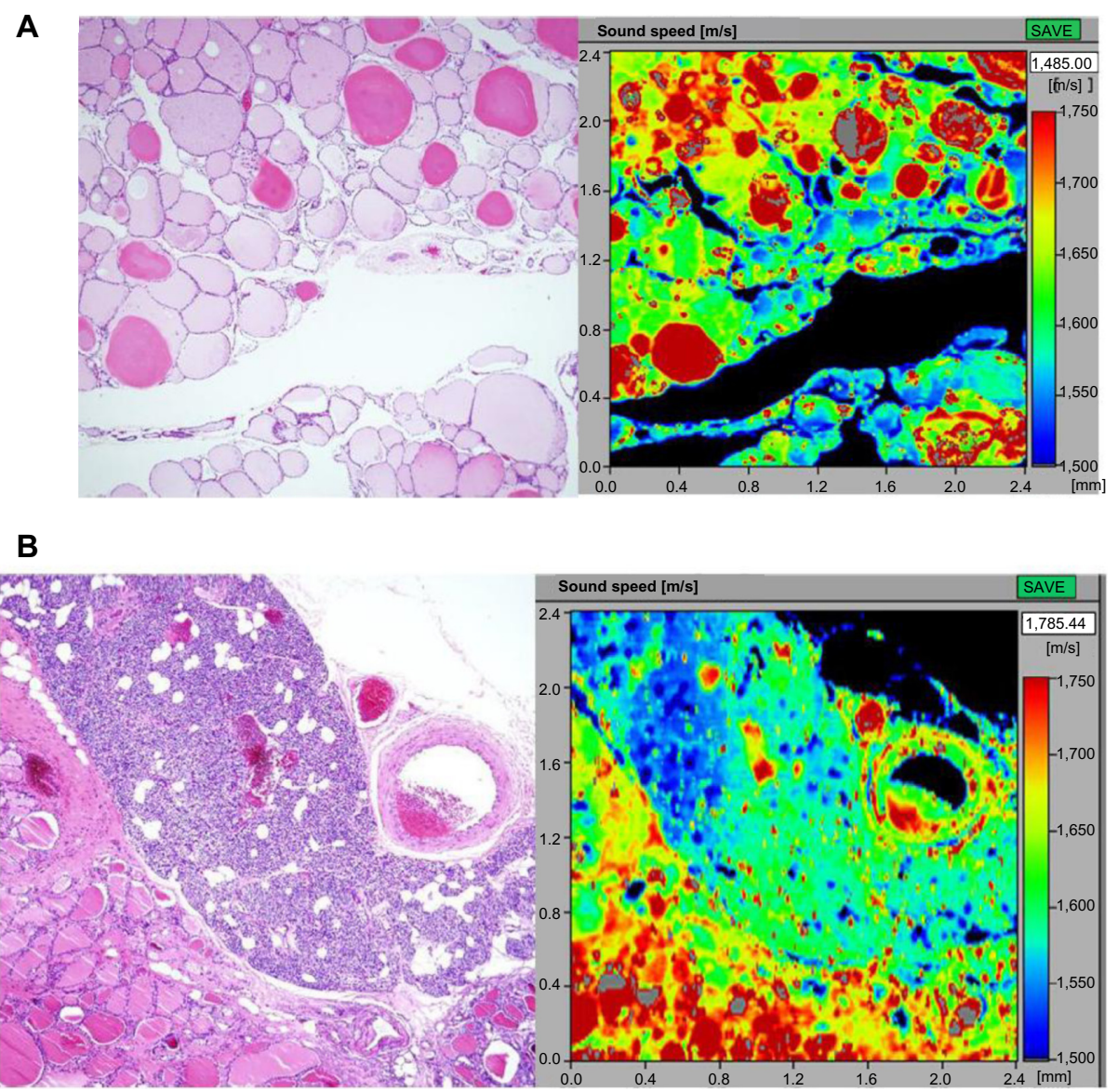

Figure 3 (A) Normal thyroid follicles. Round follicles contain colloids of various densities. Condensed colloids in hematoxylin and eosin staining (left) are shown in red with speed-of-sound $>1,750 \mathrm{~m} / \mathrm{s}$ on scanning acoustic microscope imaging (right). On the contrary, pale eosinophilic colloids appear blue-green in color on scanning acoustic microscope imaging with slower speed-of-sound (I,550-I,600 m/s). (B) Parathyroid tissue shows lower speed-of-sound at approximately I,600 m/s compared with surrounding thyroid colloids at approximately $1,700 \mathrm{~m} / \mathrm{s}$.

Abbreviation: $\mathrm{m} / \mathrm{s}$, meter/second. 
A

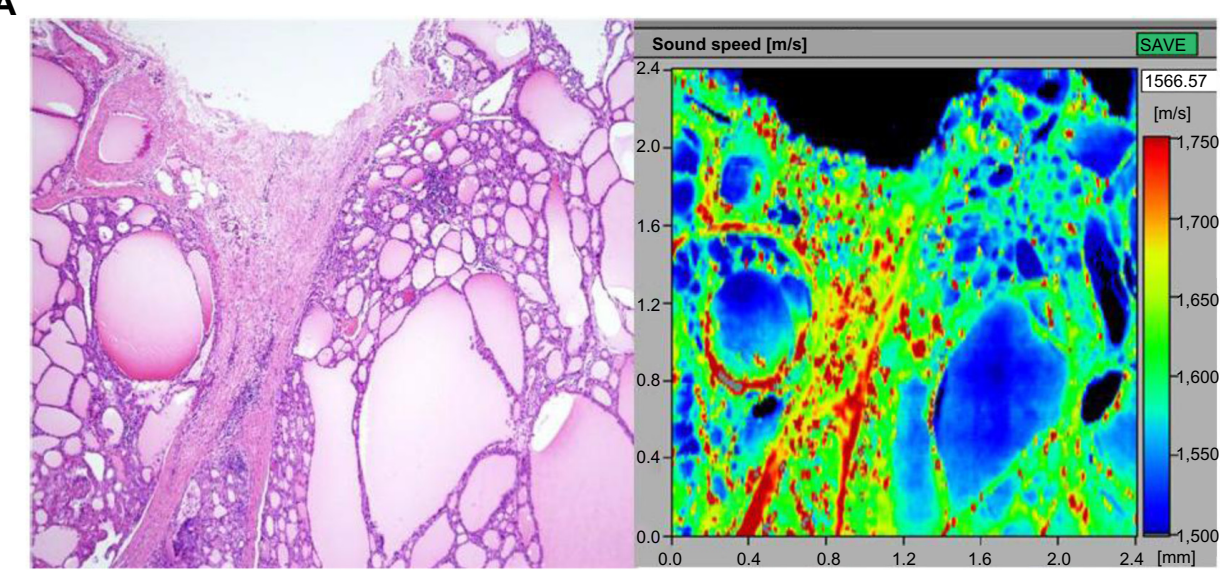

B

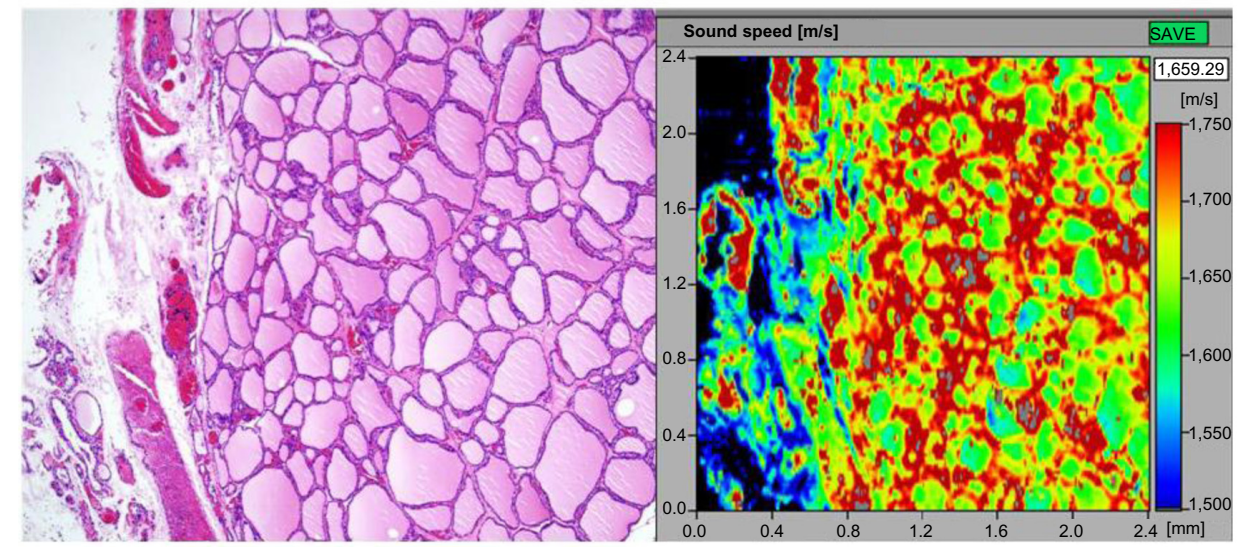

Figure 4 Thyroid of Graves' disease. (A) Elongated follicles in various sizes are present. Most colloids in the follicle have pale eosinophilia because of active secretion (left). The colloids correspond to blue in color or a speed-of-sound of approximately $1,550 \mathrm{~m} / \mathrm{s}$ on scanning acoustic microscope imaging. Fibrous septa appear red-yellow in color, which indicates a speed-of-sound of approximately I,700 m/s on scanning acoustic microscope imaging. (B) Follicles of uniform size contain light pink colloids (left) and show a green-blue color with a speed-of-sound of approximately I,600 m/s (right) on scanning acoustic microscope imaging. Stroma surrounding follicles appears faster (red color) and forms reticular structures on scanning acoustic microscope imaging.

Abbreviation: $\mathrm{m} / \mathrm{s}$, meter/second.

SAM imaging because of the fibrous background for chronic thyroiditis. Thyroid lymphomas showed a faster speed compared with lymphomas in other organs. ${ }^{5,6}$

Fibrous capsules or bands around carcinomas were split (Figures 6 and 8-10), and surrounding follicles were angular or irregular with fragmented colloids.

\section{Discussion}

Maximum resolution by a $120 \mathrm{MHz}$ transducer is theoretically equivalent to the wavelength of $12.5 \mu \mathrm{m}$, which is calculated as an SOS of $1,500 \mathrm{~m} / \mathrm{s}$ in water divided by a wave frequency of $120 \mathrm{MHz}$. Although individual cell contours were not very sharp, component thyroid structures were clearly visualized by SAM, and SAMs quality was almost equivalent to the low magnification of LM, as observed in the current results.

From the calculated results, the average SOS of the thyroid lesions commonly ranged from 1,569-1,814 m/s. Most lesions had a distinct SOS and allowed for precise SAM images equivalent to those obtained using LM. Protein or tissue fibers increased SOS according to their concentration, while epithelial cells and lymphoid tissues showed low SOS.

Major characteristic images of thyroid tumors were acquired. Thyroid tumors were divided into three groups according to SOS. Follicular adenomas/carcinomas and malignant lymphomas arising from Hashimoto's thyroiditis were included in the fast group, while the poorly differentiated carcinomas and undifferentiated carcinomas were included in the slow group. Papillary and medullary carcinomas revealing SOS between the two groups were included in the intermediate group.

Follicular adenomas/carcinomas have a conspicuous amount of colloids, which increases SOS. Malignant lymphomas of the thyroid arising from Hashimoto's thyroiditis commonly have a fibrous background. They revealed great 
A

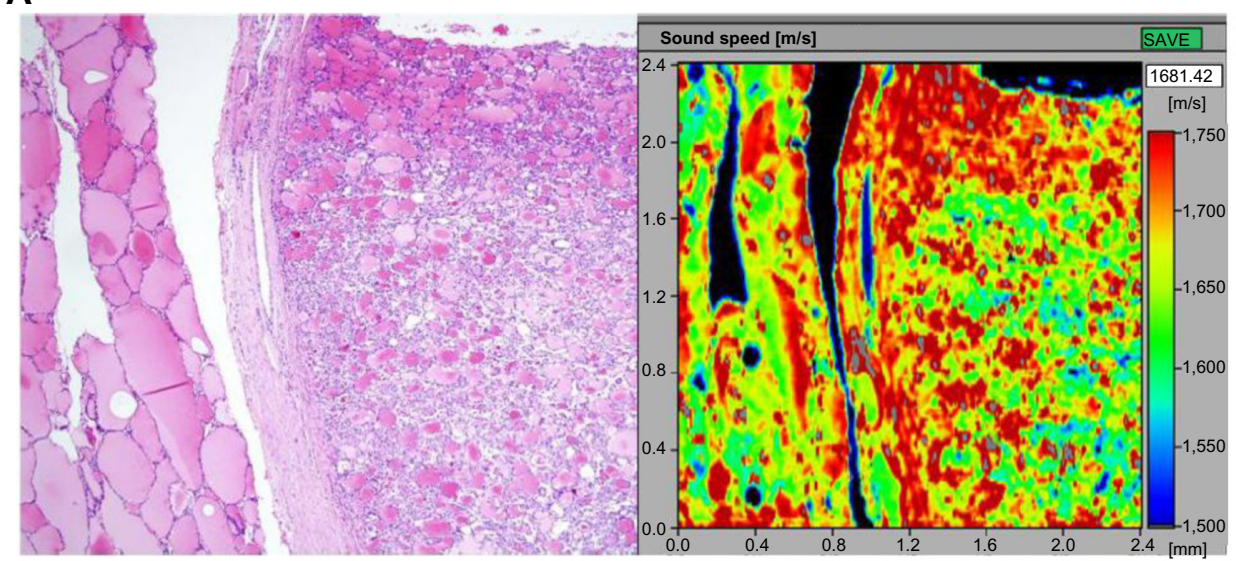

B

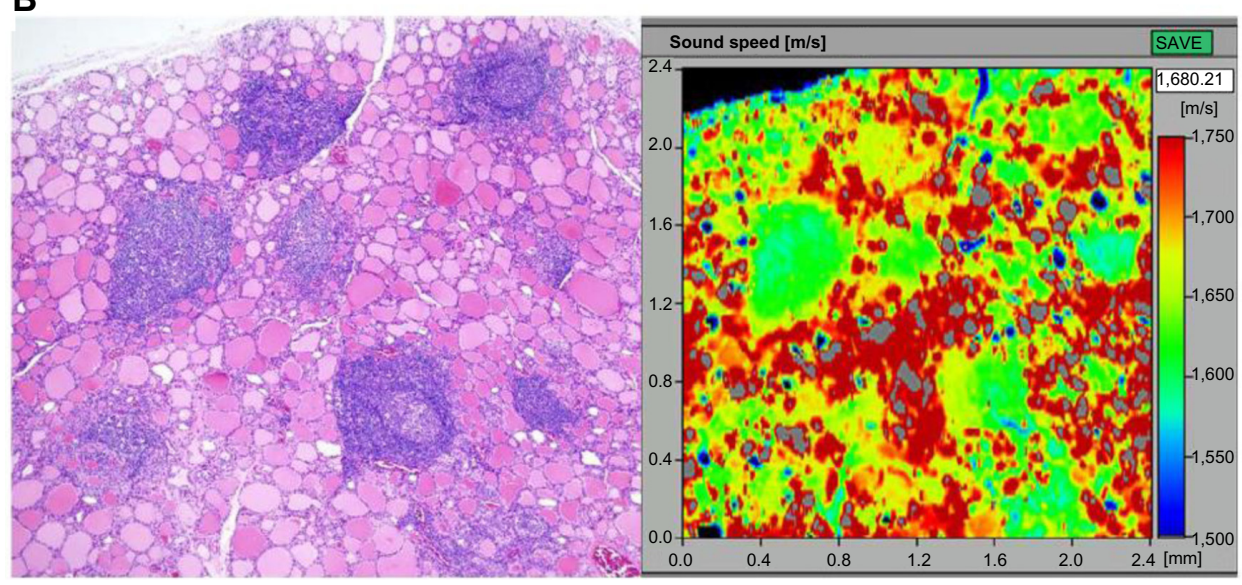

Figure 5 (A) Follicular adenoma. Adenoma consisting of microfollicles is surrounded by a fibrous septum. Microfollicles containing deeply eosinophilic colloids are red colored with a speed-of-sound $>1,750 \mathrm{~m} / \mathrm{s}$ on scanning acoustic microscope imaging. (B) Hashimoto's thyroiditis. Several lymph follicles are present among follicles with dense colloids (left). Lymph follicles correspond to green areas or a speed-of-sound of approximately I,600 m/s on scanning acoustic microscope imaging (right). Dense colloids are red colored with a speed-of-sound $>1,750 \mathrm{~m} / \mathrm{s}$.

Abbreviation: $\mathrm{m} / \mathrm{s}$, meter/second.

SOS compared with lymphomas of other organs such as the stomach $^{5}$ and lymph nodes. ${ }^{6}$

Rapidly growing tumors, such as the undifferentiated or poorly differentiated carcinomas, showed the slowest SOS, probably because of collagen-deficient stroma. From previous observations, carcinomas had slow SOS if few desmoplastic reactions occurred. ${ }^{4,5}$

Papillary carcinomas showed intermediate SOS. Although epithelial carcinoma cells themselves displayed slow SOS, fibrous cores displayed great SOS, and the average SOS approached intermediate levels. Surrounding fibrous portions with great SOS under SAM were induced by cancer invasion. If fresh frozen sections were used, surrounding follicles containing colloids showed less SOS. Therefore, desmoplastic portions with a great SOS contrasted the invasion of carcinomas, which has been indicated by conventional US features such as irregular margins and solid aspects. ${ }^{1}$
Colloid density correlates with thyroid function. Actively secreting glands present pale eosinophilic colloids with hematoxylin and eosin staining, while resting follicles show deep eosinophilic colloids. ${ }^{9}$ Dense colloids appear in atrophic small follicles, such as those observed in Hashimoto's thyroiditis, and compressed follicles are present around the main tumors, while thin colloids are commonly viewed in hyperfunctioning follicles, such as those in Graves' disease. SAM imaging provided digital SOS data of colloids, and they were parallel with protein concentration. Because SAM supplied comparable digital values of average colloid concentration, it may predict the state of thyroid function.

Low-resolution imaging is a major limitation of SAM compared with LM imaging. LM is superior to SAM for routine diagnosis of neoplastic lesions. However, SAM can provide digital data of colloid concentrations, which are statistically comparable among lesions for predicting thyroid function. 


\section{A}

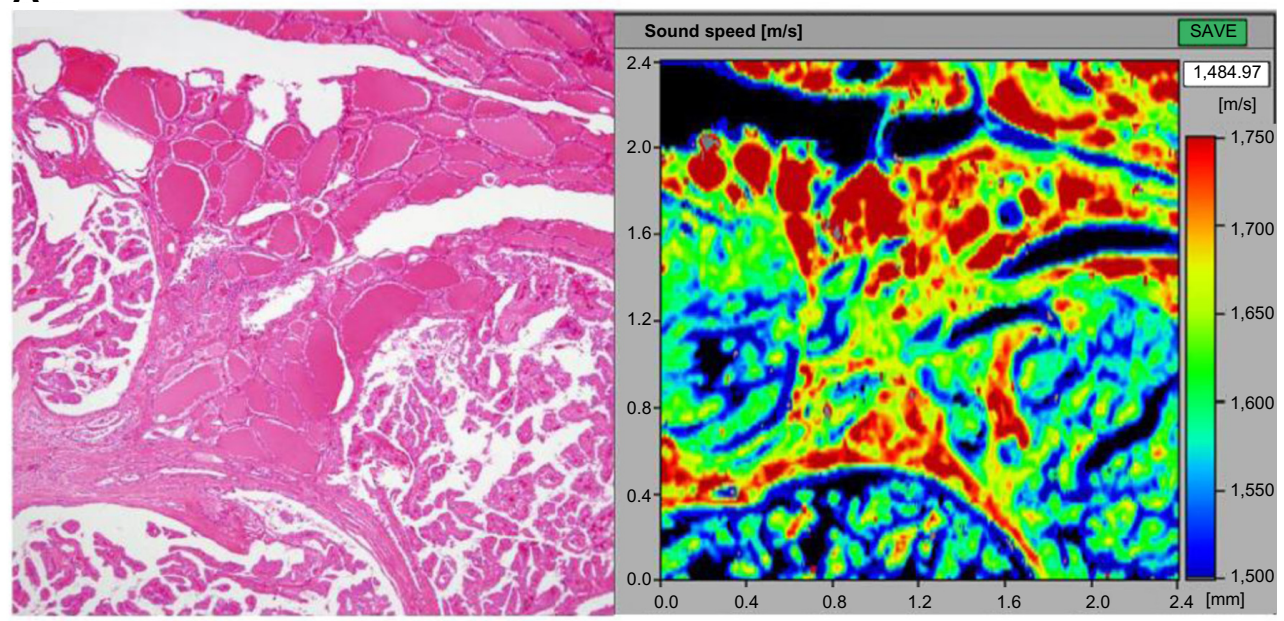

B

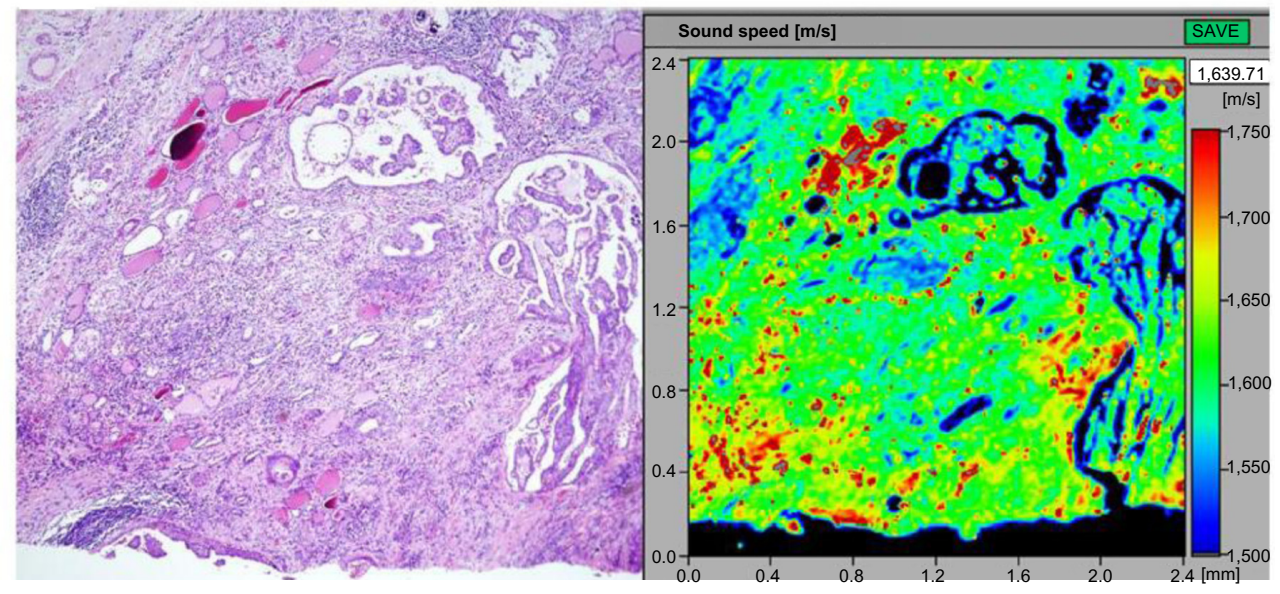

Figure 6 (A) Papillary carcinomas. Papillary carcinomas consist of thin elongating branching cores. On scanning acoustic microscope imaging, carcinomas appear blue-green in color at a speed-of-sound of approximately I,600 m/s, and fibrous cores display yellow-red dots at approximately I,680 m/s. Surrounding follicles are broken with irregular shaped colloids by invasion. (B) Papillary carcinoma invasion into surrounding normal thyroid tissues. Invasive areas with desmoplastic reactions (lower half) show greater speed-of-sound than papillary carcinoma areas (I,650 versus I,600 m/s, respectively). Red-colored spots at a speed-of-sound of approximately $1,750 \mathrm{~m} / \mathrm{s}$ on scanning acoustic microscope imaging correspond to fragmented colloids by invasion.

Abbreviation: $\mathrm{m} / \mathrm{s}$, meter/second.

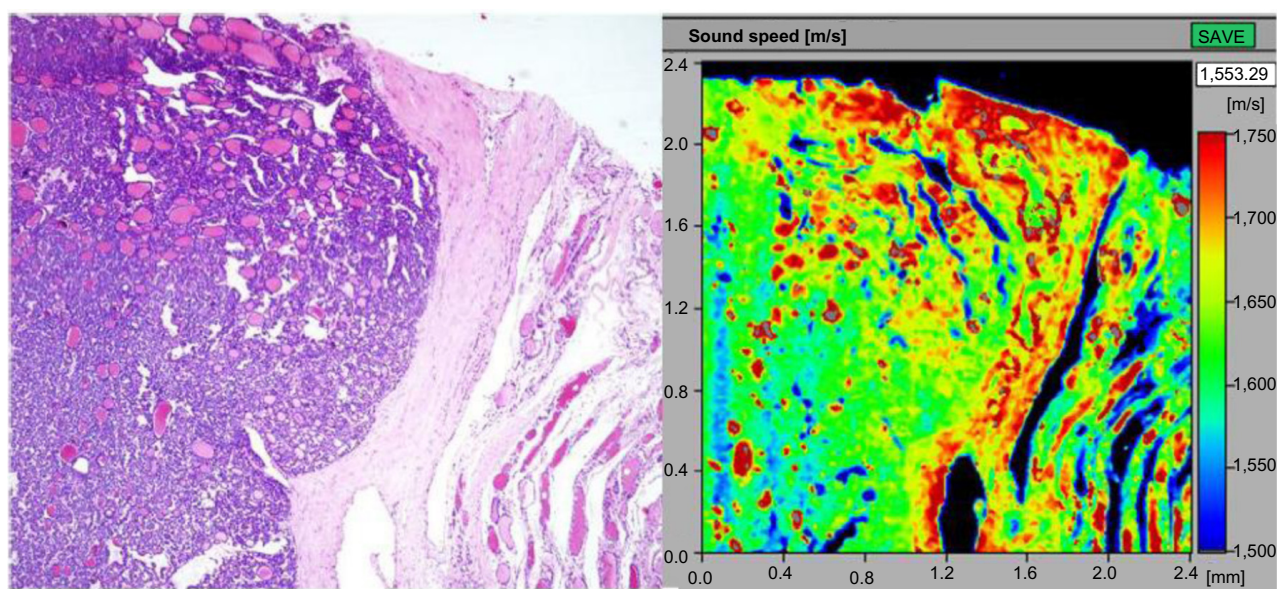

Figure 7 Follicular carcinomas. A solid tumor consisting of microfollicles (left half) compresses surrounding normal follicles with fibrous septum. Condensed colloids are red colored with a speed-of-sound $>1,750 \mathrm{~m} / \mathrm{s}$ on scanning acoustic microscope imaging, while follicles without colloids or pale colloids (left lower quadrant) are green-blue in color at approximately I,570 m/s.

Abbreviation: $\mathrm{m} / \mathrm{s}$, meter/second. 


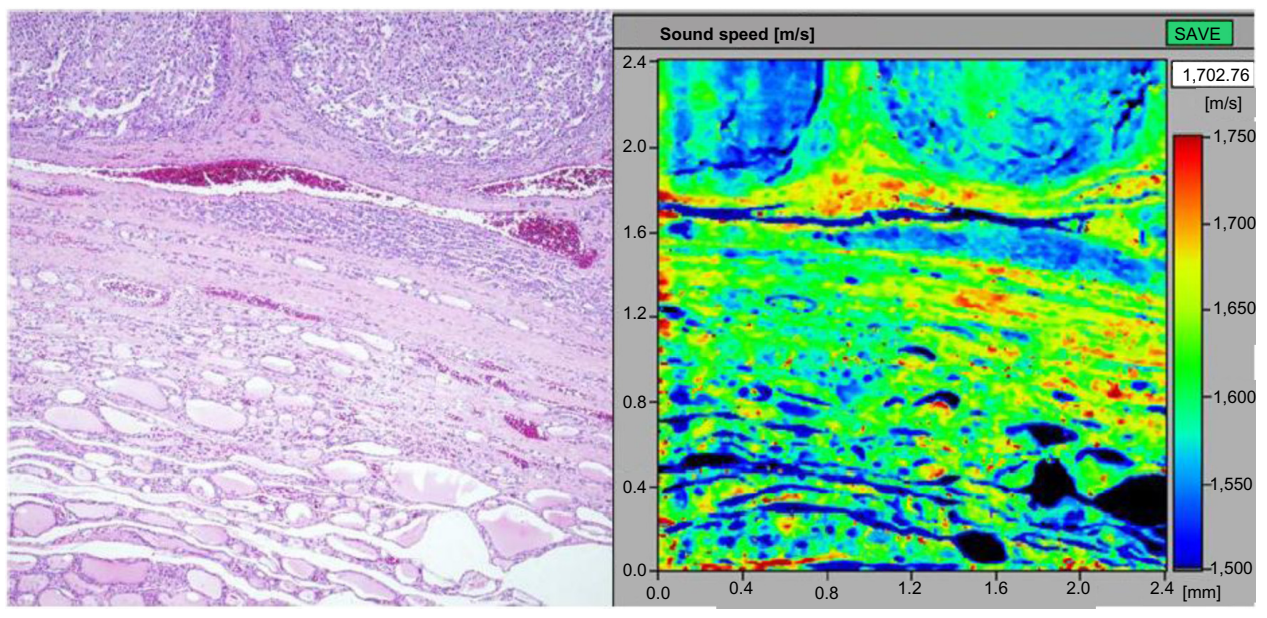

Figure 8 Poorly differentiated carcinomas. Two nodules at the upper half showed poorly differentiated carcinomas that penetrate the fibrous capsule and invade the vessels. Carcinoma portions appear blue-green in color at approximately $1,575 \mathrm{~m} / \mathrm{s}$ on scanning acoustic microscope imaging, while fibrous capsules and blood contents are yellow-orange in color with a speed-of-sound of approximately $1,700 \mathrm{~m} / \mathrm{s}$. Fibrous capsules are split by tumor invasion.

Abbreviation: $\mathrm{m} / \mathrm{s}$, meter/second.

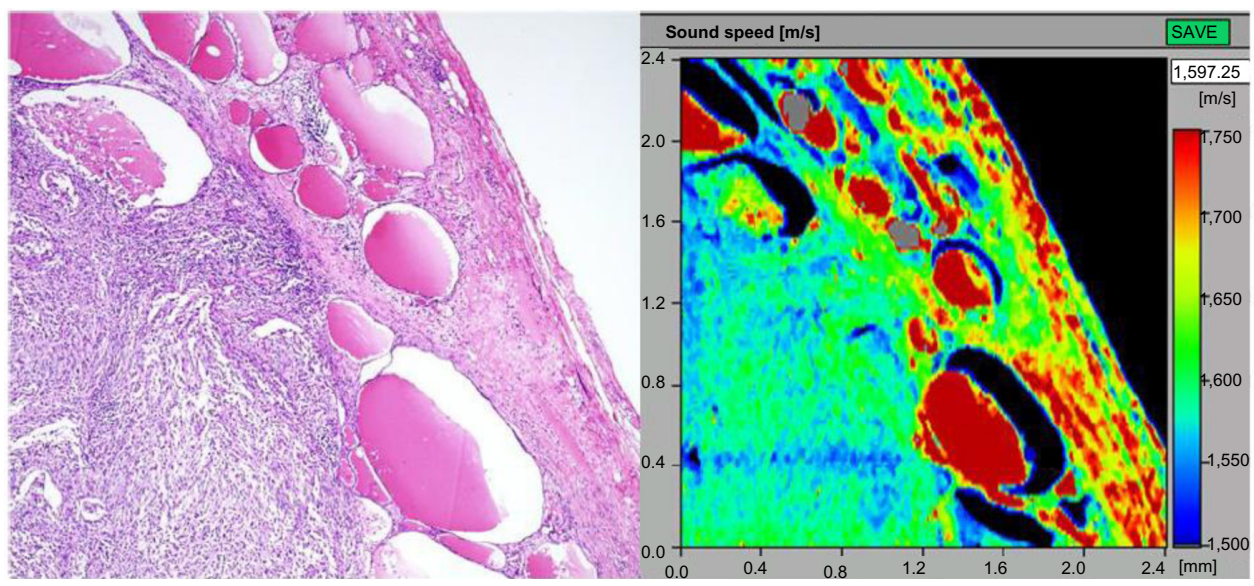

Figure 9 Undifferentiated carcinomas. An undifferentiated carcinoma consisting of spindle cells invades into the surrounding thyroid follicles. Some colloids surrounding the carcinoma are broken down into small pieces. The carcinoma portions are green-blue in color at approximately I,570 m/s on scanning acoustic microscope imaging. Compressed follicles have condensed colloids with greater speed-of-sound $(>1,750 \mathrm{~m} / \mathrm{s})$.

Abbreviation: $\mathrm{m} / \mathrm{s}$, meter/second.

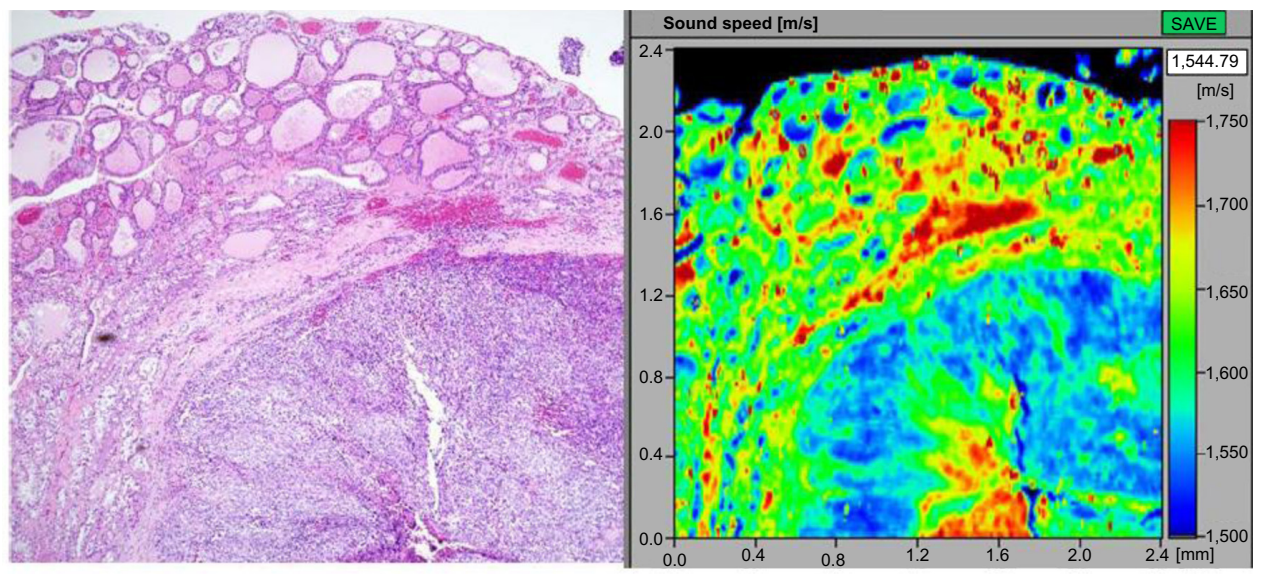

Figure 10 Medullary carcinomas. A nodule of medullary carcinoma is blue-green in color at approximately I,550 m/s on scanning acoustic microscope imaging. The central blood-rich area is red-orange in color with the same speed-of sound (approximately $1,750 \mathrm{~m} / \mathrm{s}$ ) as the perinodular blood in the vessel. Fibrous bands around the carcinoma are split, and surrounding follicles show small angular shapes.

Abbreviation: $\mathrm{m} / \mathrm{s}$, meter/second. 


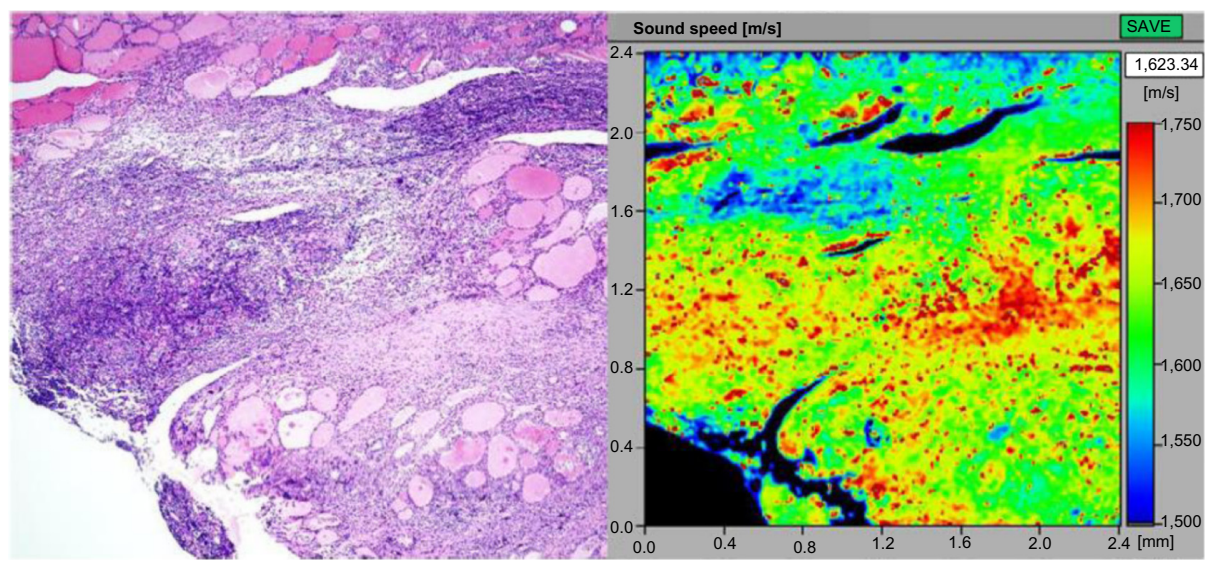

Figure II Malignant lymphoma arising from Hashimoto's thyroiditis. Several lymphoma cells penetrate through thyroid follicles and destroy them. Several tiny dots correspond to the fragmented red-colored colloids. Most lymphoma portions have a fibrous background because of Hashimoto's thyroiditis and are yellow-green in color at approximately $1,650 \mathrm{~m} / \mathrm{s}$ on scanning acoustic microscope imaging.

Abbreviation: $\mathrm{m} / \mathrm{s}$, meter/second.

Moreover, the degree of stromal reactions around each neoplastic or inflammatory lesion was objectively demonstrable by comparing SOS of the reactions.

To date, SAM imaging requires additional improvements such as improvement in resolution and overcoming the limitation of the observation area. SAM imaging is a novel method and is now being developed for application to pathological diagnosis. High-resolution and user-friendly equipment will provide histology at the cellular level that is equal or superior to LM. As another method of ultrasound utilization, SAM can use sound reflected from the surface, as used in echography. Acoustic impedance is different among tissues and lesions. To date, it is technically difficult to correctly catch reflected sound from the tissue surface, but it will be possible in the future. US imaging is now widely used in thyroid echography. The SAM results do not overcome practical LM imaging but may provide helpful information for experimental trials and future usage of ultrasound for histological diagnoses.

\section{Acknowledgments}

The authors thank Dr K Kobayashi (Honda Electronics Co, Ltd, Toyohashi, Japan) for his technical support and advice on scanning acoustic microscopy and Ms Y Kawabata and N Suzuki for preparing the tissue sections. The authors would like to thank Enago (http://www.enago.jp) for the English language review.

Pathology and Laboratory Medicine International

\section{Publish your work in this journal}

Pathology and Laboratory Medicine International is a peer-reviewed, open access journal focusing on innovative basic research and translational research related to pathology or human disease. The journal includes original research, updates, case reports, reviews and commentaries on current controversies. The Academic Sponsor
This work was supported by Japan Society for the Promotion of Science KAKENHI, Grant Number Scientific Research (c) 24590445.

\section{Disclosure}

The authors report no conflicts of interest in this work.

\section{References}

1. Rago T, Vitti P. Role of thyroid ultrasound in the diagnostic evaluation of thyroid nodules. Best Pract Res Clin Endocrinol Metab. 2008;22(6): 913-928.

2. Miura K, Yamamoto S. Histological imaging from speed-of-sound through tissues by scanning acoustic microscopy (SAM). Protocol Exchange. Epub April 15, 2013.

3. Wells PNT. Velocity, absorption and attenuation in biological materials In: Biomedical Ultrasonics. London: Academic Press; 1977:110-137.

4. Miura K, Yamamoto S. Pulmonary imaging with a scanning acoustic microscope discriminates speed-of-sound and shows structural characteristics of disease. Lab Invest. 2012;92(12):1760-1765.

5. Miura K, Yamamoto S. Histological imaging of gastric tumors by scanning acoustic microscope. British Journal of Applied Science and Technology. 2013;4(1):1-17.

6. Miura K, Nasu H, Yamamoto S. Scanning acoustic microscopy for characterization of neoplastic and inflammatory lesions of lymph nodes. Sci Rep. 2013;3:1255.

7. DeLellis R, Lloyd R, Heitz P, Eng C. World Health Organization Classification of Tumours: Pathology and Genetics of Tumours of Endocrine Organs. Lyon: IARC Press; 2004.

8. Maev RG. Acoustic properties of biological tissues and effect on the image contrast. In: Acoustic Microscopy, Fundamentals and Application. Weinheim: Wiley-VCH; 2008:212-213.

9. Mills SE, editor. Histology for Pathologists. 3rd ed. Lippincott Williams and Wilkins; 2007;44:1132-1133.

of this journal is the Chinese American Pathology Association (CAPA). The manuscript management system is completely online and includes a very quick and fair peer-review system. Visit http://www.dovepress.com/testimonials.php to read real quotes from published authors

Submit your manuscript here: http://www.dovepress.com/pathology-and-laboratory-medicine-international-journal 